Dieses Dokument ist eine Zweitveröffentlichung (Postprint) /

This is a self-archiving document (accepted version):

Halid Mulaosmanovic, Evelyn T. Breyer, Thomas Mikolajick, Stefan Slesazeck

Recovery of Cycling Endurance Failure in Ferroelectric FETs by SelfHeating

Erstveröffentlichung in / First published in:

IEEE Electron Device Letters. 2019, 40(2), S. 216-219 [Zugriff am: 18.08.2021]. IEEE. ISSN 15580563.

DOI: https://doi.org/10.1109/LED.2018.2889412

Diese Version ist verfügbar / This version is available on:

https://nbn-resolving.org/urn:nbn:de:bsz:14-qucosa2-767758 


\title{
Recovery of Cycling Endurance Failure in Ferroelectric FETs by Self-Heating
}

\author{
Halid Mulaosmanovic $^{(0)}$, Evelyn T. Breyer, Thomas Mikolajick ${ }^{\circledR}$, Senior Member, IEEE, \\ and Stefan Slesazeck
}

\begin{abstract}
This letter investigates the impact of selfheating on the post-cycling functionality of a scaled hafnium oxide-based ferroelectric field-effect transistor (FeFET). The full recovery of FeFET switching properties and data retention after the cycling endurance failure is reported. This is achieved by damage annealing through localized heating, which is intentionally induced by a large current flow through the drain (source)-body $p-n$ junctions. The results highlight that the local thermal treatments could be exploited to extend the cycling endurance of FeFETs.
\end{abstract}

Index Terms-Ferroelectric field-effect transistor (FeFET), hafnium oxide, cycling endurance, self-heating, failure recovery.

\section{INTRODUCTION}

$\mathbf{T}$ HE ferroelectric field-effect transistor (FeFET) based on ferroelectric hafnium oxide $\left(\mathrm{HfO}_{2}\right)$ is currently being considered as a possible candidate for embedded nonvolatile memory [1]. Moreover, its particular switching behavior has recently inspired several proposals for unconventional applications, including artificial neurons [2] and synapses [3], [4], ferroelectric oscillators [5], logic-in-memory devices [6], [7] and random number generators [8]. However, FeFETs have a rather limited cycling endurance, which typically ranges between $10^{4}$ and $10^{7}$ cycles [9]-[13], depending on the structure, fabrication and cycling process. The related degradation has been mainly attributed to the wear-out of the interface between $\mathrm{HfO}_{2}$ and silicon, driven by the charge trapping upon the bipolar voltage cycling [14]. Such a limitation might be the main bottleneck for the success of some of the abovementioned applications and, thus, strategies for increasing the number of switching cycles are needed.

Recently, local annealing methods have been investigated for improving or restoring the degraded performance of logic and memory devices. They rely on damage healing by the

Manuscript received December 5, 2018; accepted December 19, 2018. Date of publication December 24, 2018; date of current version January 31,2019 . This work was financially supported by the European Fund for regional Development EFRD and the Free State of Saxony, Europe supports Saxony. The review of this letter was arranged by Editor A. Naeemi. (Corresponding author: Halid Mulaosmanovic.)

H. Mulaosmanovic, E. T. Breyer and S. Slesazeck are with NaMLab gGmbH, 01187 Dresden, Germany (e-mail: halid.mulaosmanovic@ namlab.com).

T. Mikolajick is with NaMLab gGmbH, 01187 Dresden, Germany, and also with the Chair of Nanoelectronic Materials, Technical University Dresden, 01062 Dresden, Germany.

Color versions of one or more of the figures in this letter are available online at http://ieeexplore.ieee.org.

Digital Object Identifier 10.1109/LED.2018.2889412
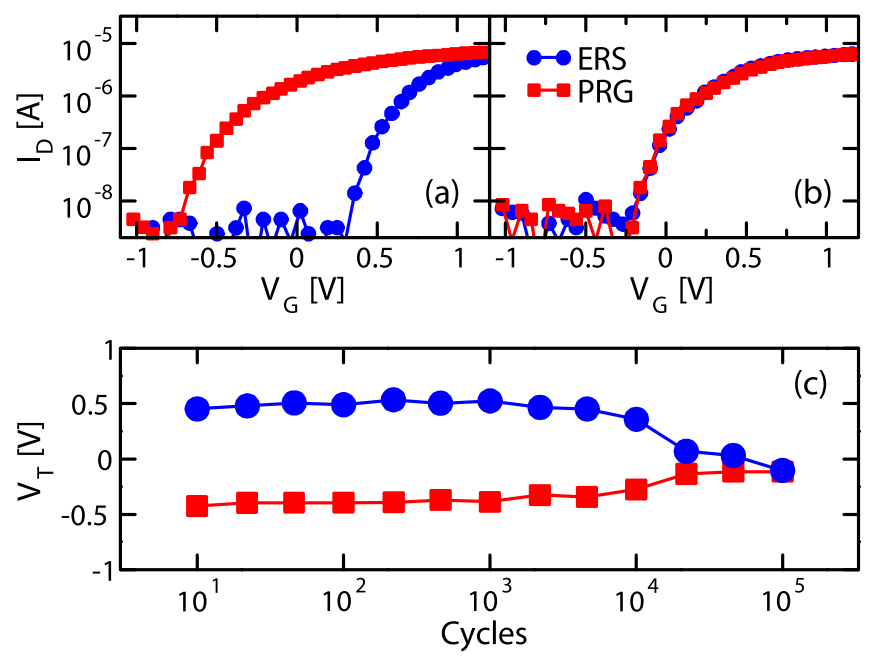

Fig. 1. Effect of the bipolar cycling with PRG: $\left(V_{P}=3.2 \mathrm{~V}, 1 \mu \mathrm{s}\right)$, and ERS: $\left(V_{N}=-4.5 \mathrm{~V}, 1 \mu \mathrm{s}\right)$. $I_{D^{-}} V_{G}$ curves for the two states after (a) $10^{2}$ cycles, and (b) $10^{5}$ cycles. (c) $V_{T}$ evolution with cycling. The curves are measured at drain voltage $V_{D}=100 \mathrm{mV}$ by a fast $V_{G}$ sweep, which limits the lower resolution to $10 \mathrm{nA}$. $V_{T}$ is extracted at constant drain current $I_{D}=0.1 \mu \mathrm{A} \cdot \mathrm{W} / \mathrm{L}$.

localized heat generation, e.g. induced by the current flow through a double-ended word-line in NAND flash memory [15] and gate-all-around MOSFETs [16], the $p-n$ junction forward current [17], the die-stacked heaters [18], [19] and monolithically integrated micro-heaters [20].

In this letter, we explore the impact of self-heating on the post-cycling functionality and endurance of scaled FeFETs. By locally inducing Joule heating, which in our experiments is generated upon intentionally forward-biasing the drain (source)-body junction, a full recovery of the FeFET switching and data retention after the cycling endurance failure is reported. Finally, the adoption of this method for enhancing the device endurance is discussed.

\section{Experimental Results}

Experiments were performed on FeFETs fabricated using the $28 \mathrm{~nm}$ high-k metal gate process flow in a gate-first approach, as described in [21]. The devices are characterized by a $1.2 \mathrm{~nm}$ thick SiON interface layer and a $10 \mathrm{~nm}$ thick $\mathrm{Si}: \mathrm{HfO}_{2}$ layer. The channel length $(L)$ and width $(W)$ of the transistor are $30 \mathrm{~nm}$ and $80 \mathrm{~nm}$, respectively.

To set the FeFET into low (high) threshold voltage $\left(V_{T}\right)$ state, a sufficiently large positive $V_{P}$ (negative $V_{N}$ ) gate pulse 

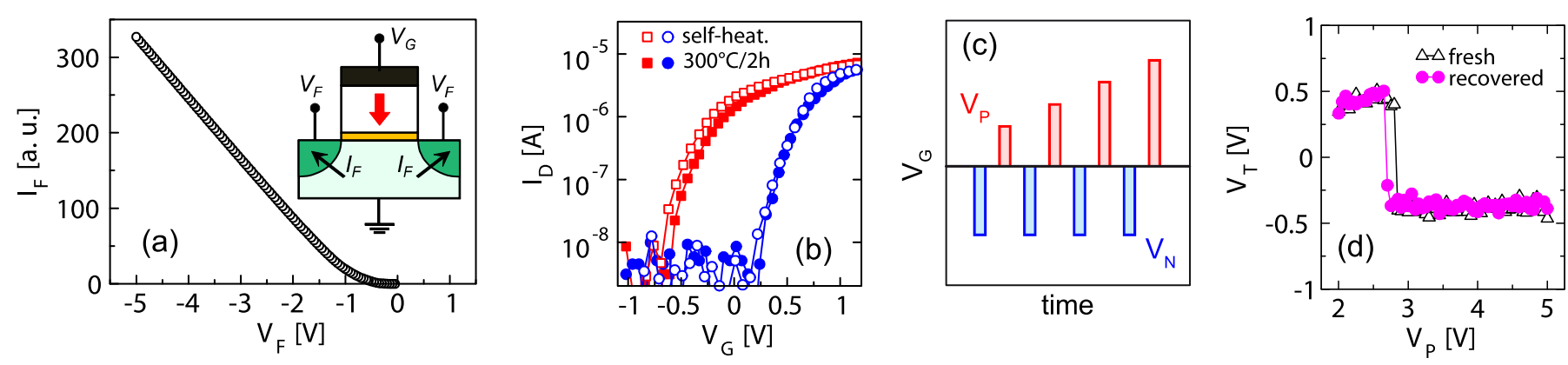

Fig. 2. (a) Forward-bias current $I_{F}$ through drain-body $p$ - $n$ junction, measured by sweeping the drain voltage $V_{F}$; (b) $I_{D}-V_{G}$ curves of a recovered device for two types of thermal treatment: self-heating under $V_{F}$ pulses and baking in the oven; (c) pulsing scheme for investigating the switching from low- to high- $V_{T}$ state. After each pulse, the corresponding $I_{D}-V_{G}$ is collected; (d) Switching of the fresh and recovered device shown as $V_{T}$ vs. $V_{P}$, obtained with the waveform shown in (c).

has to be applied. In this way, a program, PRG (erase, ERS) transition is induced.

Fig. 1 shows the so obtained PRG and ERS $I_{D}-V_{G}$ curves and their evolution with the increasing number of endurance cycles. The memory window and the $V_{T}$ values relative to the two logic states remain almost constant up to $10^{4}$ cycles. However, further cycling rapidly leads to performance degradation and to the complete collapse of the memory window after $10^{5}$ cycles (endurance failure, Fig. 1b).

Interestingly, by forward biasing the drain-body and sourcebody junction and performing a DC sweep at room temperature as indicated in Fig. 2a, such a post-cycled and nonfunctional FeFET fully regains the switching property and its memory window. The same effect is achieved in the pulsed regime as well, where $V_{F}$ pulses are simultaneously applied at source and drain, while keeping the bulk grounded. For instance, $(-3 \mathrm{~V}, 1 \mathrm{~s})$, and $(-4 \mathrm{~V}, 100 \mathrm{~ms})$ were found to be suited combinations of $V_{F}$ amplitude and duration, where the latter pulse setting will be used in the following discussion. Fig. $2 \mathrm{~b}$ shows the restored PRG and ERS $I_{D}-V_{G}$ curves of the device of Fig. 1 after the pulsed treatment. The recovery effect is attributable to the local Joule heating generated by the large forward current in the $p-n$ junction, which heals out the damage at the SiON interface induced by cycling. A similar healing phenomenon has been previously reported for recovering the hot-carrier injection induced damage in short channel logic devices [17]. It should be mentioned that the recovery after cycling can be alternatively achieved just by externally annealing the device at elevated temperatures (e.g. by baking in the oven at $300^{\circ} \mathrm{C}$ for 2 hours, as shown for comparison in Fig. 2b). Even though this is the first experimental evidence for the recovery in FeFETs through an external thermal treatment, such an off-chip technique has a very limited applicability. On the other hand, the recovery could not be achieved by applying a write pulse (ERS or PRG) of an excessively large amplitude and/or duration, as has been reported for some sorts of resistive switching devices [22]. This would, namely, lead to the irreversible hard breakdown of the gate-stack.

Although the recovered device displays a proper memory window, it might be affected by an undesired shift of the PRG and ERS $V_{T}$ levels and/or a significant change of the switching response. To verify this, we have performed the analysis of the switching voltage of the pre-cycled and of the recovered device by using the gate waveform shown in Fig. 2c. As can
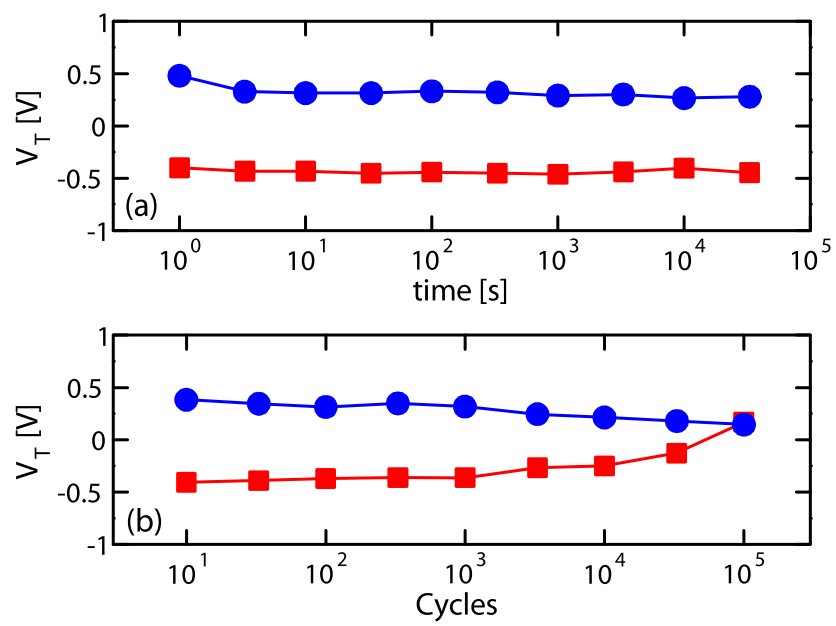

Fig. 3. (a) Retention at room temperature and (b) cycling endurance for the two $V_{T}$ states of the recovered device.

be observed in Fig. 2d, the switching voltage, defined as the gate pulse $V_{P}$ for which an abrupt transition from high- to low- $V_{T}$ state occurs [21], is almost unchanged and differs by only $100 \mathrm{mV}$. Note that this value is within the cycle-to-cycle statistical dispersion range, which is normally displayed by these devices [8], [21]. In addition, both PRG and ERS $V_{T}$ levels are preserved after the recovery.

To gain more insight into the performance of the recovered device, we performed data retention and cycling endurance experiments. Fig. 3a shows that the FeFET exhibits a very stable retention of the two logic states, similar to the one of the fresh device [23]. The endurance experiment of Fig. 3b shows that the recovered FeFET can still withstand between $10^{4}$ and $10^{5}$ switching cycles prior to the failure. However, in contrast to the fresh device, the degradation sets in for both states already after $10^{3}$ cycles. This might imply that $(-4 \mathrm{~V}$, $100 \mathrm{~ms}$ ) is not the optimal healing condition and further tuning of $V_{F}$ parameters is needed.

The beneficial effect of the local heating could be exploited to extend the FeFET endurance. A conceivable approach in this regard might be to periodically apply self-heating pulses before a significant degradation sets in, for instance, after a limited number of switching cycles that are not capable of causing the endurance failure (e.g. $N \leq 10^{4}$ ). Fig. 4b shows 
(a)
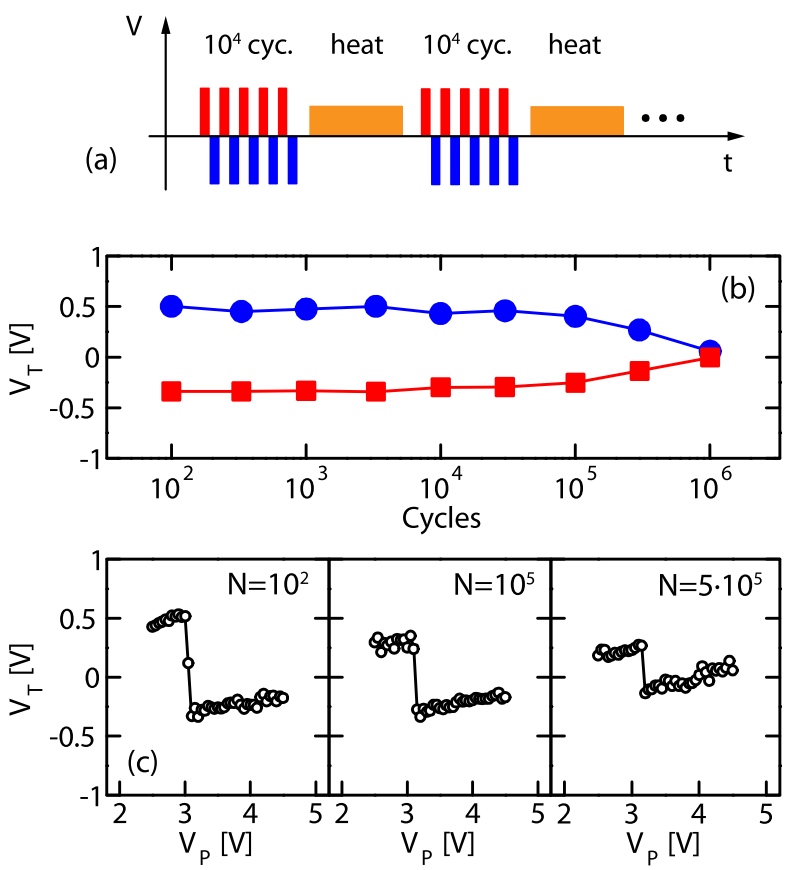

Fig. 4. (a) Pulsing scheme for extending the cycling endurance. A self-heating $V_{F}$ pulse at drain and source terminal is interposed between blocks of $10^{4}$ switching cycles. (b) $V_{T}$ evolution with the cycling scheme in (a); (c) switching behavior after $10^{2}, 10^{5}$ and $5 \cdot 10^{5}$ bipolar cycles.

the results of one such experiment, where the $V_{F}$ pulses are interposed between blocks of $10^{4}$ bipolar cycles, as depicted schematically in Fig. 4a. The endurance indeed improves and extends for more than a factor of 10 with respect to the result in Fig. 1. Moreover, Fig. 4c shows that the proper and abrupt switching could be detected even after $10^{5}$ cycles as well as after $5 \cdot 10^{5}$ cycles, though with a smaller memory window.

However, although the results clearly confirm the benefit of adopting the self-heating pulses, this approach cannot be used to indefinitely extend the cycling endurance in FeFETs. Indeed, the memory window collapses before reaching $10^{6}$ cycles, after which the device cannot be recovered anymore. This might be explained by assuming that the rate of defect generation under $10^{4} \mathrm{PRG} / \mathrm{ERS}$ cycles is larger than the healing rate upon a self-heating pulse, which inevitably induces degradation. In fact, the positive and the negative switching pulses approximately correspond to the energy band diagrams of the gate stack depicted in Fig. 5a and $5 \mathrm{~b}$, respectively. Owing to the large difference in dielectric constant between $\mathrm{HfO}_{2}$ and $\mathrm{SiON}$, a large portion of the applied $V_{G}$ drops across the thin SiON layer. While this favors a significant tunneling of electrons during PRG (Fig. 5a) and holes during ERS (Fig. 5b), the high interfacial field stress induces the generation of defects and the consequent rapid degradation of SiON (Fig. 5c) [14]. Since the created defects act as traps for the injected carriers, the charge trapping drastically increases at this point. The so trapped charge counteracts or even impedes the ferroelectric switching, which causes the collapse of the memory window after $10^{5}$ cycles without healing pulses (Fig. 5c). Nevertheless, this failure is recoverable with $V_{F}$ pulses (Fig. 5d), since the defects are located in the proximity of the generated heat. However, further cycling $\left(N>10^{5}\right)$ induces the generation and (a)

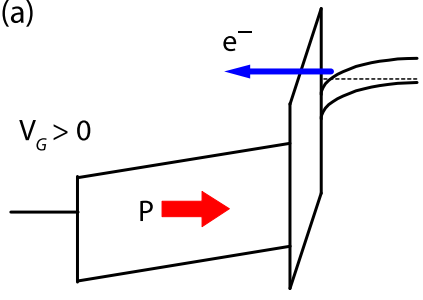

$\mathrm{TiN}$

Si: $\mathrm{HfO}_{2} \quad \mathrm{SiON}$

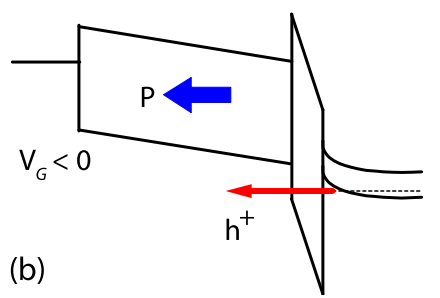

(c)

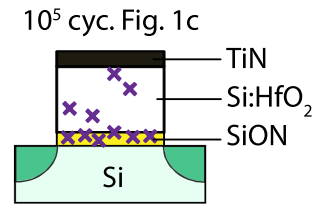

(d) during healing Fig. $2 \mathrm{~b}$

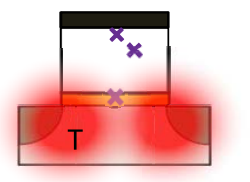

(e)

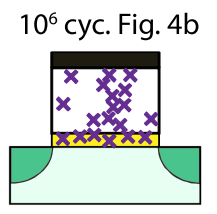

Fig. 5. Energy band diagrams of the gate stack during (a) PRG and (b) ERS operation. Only the predominant carrier injection flows are illustrated; additional tunneling paths exist as well. (c) - (e) Schematic illustration of the generation and healing of defects upon cycling and selfheating, respectively. Scenarios for (c) - (d) the recoverable and (e) the permanent breakdown.

redistribution of defects deeper in the $\mathrm{HfO}_{2}$ bulk [24], which cannot be easily healed out. This might cause the formation of defect percolation paths (Fig. 5e), which eventually leads to the hard breakdown [24].

Nevertheless, the results shown here might open up a new path for using the local annealing to boost the endurance. To achieve excellent cycling doses (e.g. the ones shown by Sakai et al. for perovskite based FeFETs [25]), further optimization and/or adoption of alternative heating methods might be taken into consideration. For instance, the heating through double-ended word-lines [15] or monolithically integrated micro-heaters [20] could be a possible option. Special attention should be given to the device geometry as well, which greatly influences the heat transfer [26]. Finally, these thermal approaches could possibly complement other, already proposed strategies for endurance enhancement [27].

\section{CONCLUSION}

We have explored the impact of the local heating on the performance of a cycled FeFET device. Joule heating, which is intentionally induced by forward-biasing the drain (source)bulk $p$ - $n$ junctions, almost completely restores the FeFET functionality after the endurance failure. Moreover, external annealing (e.g. baking in the oven) leads to the same outcome. The recovered device preserves the switching behavior, i.e. switching voltage and abruptness of the switching transitions, threshold levels for the two states, and data retention. A pulsing scheme for extending the endurance has been proposed, which indeed shows the improvement of more than a factor of 10. Further optimization and adopting of alternative heating methods might lead to more significant improvements.

\section{ACKNOWLEDGMENTS}

The authors would like to thank GlobalFoundries Dresden, Germany, for experimental samples and support. 


\section{REFERENCES}

[1] M. Trentzsch, S. Flachowsky, R. Richter, J. Paul, B. Reimer, D. Utess, S. Jansen, H. Mulaosmanovic, S. Müller, S. Slesazeck, J. Ocker, M. Noack, J. Müller, P. Polakowski, J. Schreiter, S. Beyer, T. Mikolajick, and B. Rice, "A $28 \mathrm{~nm}$ HKMG super low power embedded NVM technology based on ferroelectric FETs," in IEDM Tech. Dig., Dec. 2016, pp. 11.5.1-11.5.4, doi: 10.1109/IEDM.2016.7838397.

[2] H. Mulaosmanovic, E. Chicca, M. Bertele, T. Mikolajick, and S. Slesazeck, "Mimicking biological neurons with a nanoscale ferroelectric transistor," Nanoscale, vol. 10, no. 46, pp. 21755-21763, Nov. 2018, doi: $10.1039 / \mathrm{C} 8 \mathrm{NR} 07135 \mathrm{G}$.

[3] H. Mulaosmanovic, J. Ocker, S. Müller, M. Noack, J. Müller, P. Polakowski, T. Mikolajick, and S. Slesazeck, "Novel ferroelectric FET based synapse for neuromorphic systems," in Proc. Symp. VLSI Technol.(VLSIT), Jun. 2017, pp. T176-T177, doi: 10.23919/VLSIT.2017.7998165.

[4] M. Seo, M. H. Kang, S. B. Jeon, H. Bae, J. Hur, B. C. Jang, S. Yun, S. Cho, W.-K. Kim, M.-S. Kim, K.-M. Hwang, S. Hong, S.-Y. Choi, and Y.-K. Choi, "First demonstration of a logic-process compatible junctionless ferroelectric FinFET synapse for neuromorphic applications," IEEE Electron Device Lett., vol. 39, no. 9, pp. 1445-1448, Sep. 2018, doi: 10.1109/LED.2018.2852698.

[5] Z. Wang, S. Khandelwal, and A. I. Khan, "Ferroelectric oscillators and their coupled networks," IEEE Electron Device Lett., vol. 38, no. 11, pp. 1614-1617, Nov. 2017, doi: 10.1109/LED.2017. 2754138.

[6] E. T. Breyer, H. Mulaosmanovic, T. Mikolajick, and S. Slesazeck, "Reconfigurable NAND/NOR logic gates in $28 \mathrm{~nm}$ HKMG and 22 nm FD-SOI FeFET technology," in IEDM Tech. Dig., Dec. 2017, pp. 28.5.1-28.5.4, doi: 10.1109/IEDM.2017.8268471.

[7] X. Yin, A. Aziz, J. Nahas, S. Datta, S. Gupta, M. Niemier, and X. S. Hu, "Exploiting ferroelectric FETs for low-power non-volatile logic-in-memory circuits," in Proc. IEEE/ACM Int. Conf. Comput.Aided Design (ICCAD), Nov. 2016, pp. 1-8, doi: 10.1145/2966986. 2967037.

[8] H. Mulaosmanovic, T. Mikolajick, and S. Slesazeck, "Random number generation based on ferroelectric switching," IEEE Electron Device Lett., vol. 39, no. 1, pp. 135-138, Jan. 2018, doi: 10.1109/LED.2017. 2771818.

[9] S. Mueller, J. Müller, R. Hoffmann, E. Yurchuk, T. Schlösser, R. Boschke, J. Paul, M. Goldbach, T. Herrmann, A. Zaka, U. Schröder, and T. Mikolajick, "From MFM capacitors toward ferroelectric Transistors: Endurance and disturb characteristics of $\mathrm{HfO}_{2}$-based FeFET devices," IEEE Trans. Electron Devices, vol. 60, no. 12, pp. 4199-4205, Dec. 2013, doi: 10.1109/TED.2013.2283465.

[10] K. Chatterjee, S. Kim, G. Karbasian, A. J. Tan, A. K. Yadav, A. I. Khan, C. Hu, and S. Salahuddin, "Self-aligned, gate last, FDSOI, ferroelectric gate memory device with $5.5-\mathrm{nm} \mathrm{Hf}_{0.8} \mathrm{Zr}_{0.2} \mathrm{O}_{2}$, high endurance and breakdown recovery," IEEE Electron Device Lett., vol. 38 , no. 10 , pp. 1379-1382, Oct. 2017, doi: 10.1109/LED.2017. 2748992.

[11] N. Gong and T.-P. Ma, "A study of endurance issues in $\mathrm{HfO}_{2}$-based ferroelectric field effect transistors: Charge trapping and trap generation," IEEE Electron Device Lett., vol. 39, no. 1, pp. 15-18, Jan. 2018, doi: 10.1109/LED.2017.2776263.

[12] K. Ni, P. Sharma, J. Zhang, M. Jerry, J. A. Smith, K. Tapily, R. Clark, S. Mahapatra, and S. Datta, "Critical role of interlayer in $\mathrm{Hf}_{0.5} \mathrm{Zr}_{0.5} \mathrm{O}_{2}$ ferroelectric FET nonvolatile memory performance," IEEE Trans. Electron Devices, vol. 65, no. 6, pp. 2461-2469, Jun. 2018, doi: 10.1109/TED.2018.2829122.

[13] B. Zeng, W. Xiao, J. Liao, H. Liu, M. Liao, Q. Peng, S. Zheng, and Y. Zhou, "Compatibility of $\mathrm{HfN}$ metal gate electrodes with $\mathrm{Hf}_{0.5} \mathrm{Zr}_{0.5} \mathrm{O}_{2}$ ferroelectric thin films for ferroelectric field-effect transistors," IEEE Electron Device Lett., vol. 39, no. 10, pp. 1508-1511, Oct. 2018, doi: 10.1109/LED.2018.2868275.
[14] E. Yurchuk, S. Mueller, D. Martin, S. Slesazeck, U. Schroeder, T. Mikolajick, J. Müller, J. Paul, R. Hoffmann, J. Sundqvist, T. Schlosser, R. Boschke, R. van Bentum, and M. Trentzsch, "Origin of the endurance degradation in the novel $\mathrm{HfO} 2$-based $1 \mathrm{~T}$ ferroelectric non-volatile memories," in Proc. IEEE Int. Rel. Phys. Symp., Jan. 2014, pp. 2E.5.1-2E.5.5, doi: 10.1109/IRPS.2014.6860603.

[15] H.-T. Lue, P.-Y. Du, C.-P. Chen, W.-C. Chen, C.-C. Hsieh, Y.-H. Hsiao, Y.-H. Shih, and C.-Y. Lu, "Radically extending the cycling endurance of Flash memory (to $>100 \mathrm{M}$ Cycles) by using built-in thermal annealing to self-heal the stress-induced damage," in IEDM Tech. Dig., Dec. 2012, pp. 9.1.1-9.1.4, doi: 10.1109/IEDM.2012.6479008.

[16] J.-Y. Park, D.-I. Moon, M.-L. Seol, C.-K. Kim, C.-H. Jeon, H. Bae, T. Bang, and Y.-K. Choi, "Self-curable gate-all-around MOSFETs using electrical annealing to repair degradation induced from hot-carrier injection," IEEE Trans. Electron Devices, vol. 63, no. 3, pp. 910-915, Mar. 2016, doi: 10.1109/TED.2015.2513744.

[17] G.-B. Lee, C.-K. Kim, J.-Y. Park, T. Bang, H. Bae, S.-Y. Kim, S.-W. Ryu, and Y.-K. Choi, "A novel technique for curing hotcarrier-induced damage by utilizing the forward current of the PN-junction in a MOSFET," IEEE Electron Device Lett., vol. 38, no. 8, pp. 1012-1014, Aug. 2017, doi: 10.1109/LED.2017.2718583.

[18] Q. Wu, G. Dong, and T. Zhang, "A first study on selfhealing solid-state drives," in Proc. IMW, May 2011, pp. 1-4, doi: 10.1109/IMW.2011.5873201.

[19] J.-W. Han, M. Kebaili, and M. Meyyappan, "System on microheater for on-chip annealing of defects generated by hot-carrier injection, bias temperature instability, and ionizing radiation," IEEE Electron Device Lett., vol. 37, no. 12, pp. 1543-1546, Dec. 2016, doi: 10.1109/LED.2016.2616133.

[20] J.-W. Han, R. Peterson, D.-I. Moon, D. G. Senesky, and M. Meyyappan, "Monolithically integrated microheater for on-chip annealing of oxide defects," IEEE Electron Device Lett., vol. 38, no. 7, pp. 831-834, Jul. 2017, doi: 10.1109/LED.2017.2700326.

[21] H. Mulaosmanovic, J. Ocker, S. Müller, U. Schroeder, J. Müller, P. Polakowski, S. Flachowsky, R. van Bentum, T. Mikolajick, and S. Slesazeck, "Switching kinetics in nanoscale hafnium oxide based ferroelectric field-effect transistors," ACS Appl. Mater. Inter, vol. 9, no. 4, pp. 3792-3798, Jan. 2017, doi: 10.1021/acsami.6b13866.

[22] Y. Y. Chen, B. Govoreanu, L. Goux, R. Degraeve, A. Fantini, G. S. Kar, D. J. Wouters, G. Groeseneken, J. A. Kittl, M. Jurczak, and L. Altimime, "Balancing SET/RESET pulse for $>10^{10}$ endurance in $\mathrm{HfO}_{2} / \mathrm{Hf} 1 \mathrm{~T} 1 \mathrm{R}$ bipolar RRAM," IEEE Trans. Electron Devices, vol. 59, no. 12, pp. 3243-3249, Dec. 2012, doi: 10.1109/TED.2012.2218607.

[23] H. Mulaosmanovic, S. Slesazeck, J. Ocker, M. Pesic, S. Müller, S. Flachowsky, J. Müller, P. Polakowski, J. Paul, S. Jansen, S. Kolodinski, C. Richter, S. Piontek, T. Schenk, A. Kersch, C. Kunneth, R. van Bentum, U. Schroder, and T. Mikolajick, "Evidence of single domain switching in hafnium oxide based FeFETs: Enabler for multi-level FeFET memory cells," in IEDM Tech. Dig., Dec. 2015, pp. 26.8.1-26.8.3, doi: 10.1109/IEDM.2015.7409777.

[24] A. Kerber and E. A. Cartier, "Reliability challenges for CMOS technology qualifications with hafnium oxide/titanium nitride gate stacks," IEEE Trans. Device Mater. Rel., vol. 9, no. 2, pp. 147-162, Jun. 2009, doi: 10.1109/TDMR.2009.2016954.

[25] S. Sakai and R. Ilangovan, "Metal-ferroelectric-insulator-semiconductor memory FET with long retention and high endurance," IEEE Electron Device Lett., vol. 25, no. 6, pp. 369-371, Jun. 2004, doi: 10.1109/LED.2004.828992.

[26] E. Pop, S. Sinha, and K. E. Goodson, "Heat generation and transport in nanometer-scale transistors," Proc. IEEE, vol. 94, no. 8, pp. 1587-1601, Aug. 2006, doi: 10.1109/JPROC.2006.879794.

[27] J. Müller, P. Polakowski, S. Müller, H. Mulaosmanovic, J. Ocker, T. Mikolajick, S. Slesazeck, S. Flachowsky, and M. Trentzsch, "High endurance strategies for hafnium oxide based ferroelectric field effect transistor," in Proc. NVMTS, Oct. 2016, pp. 1-7, doi: 10.1109/NVMTS.2016.7781517. 